\title{
Die Thematisierung religiöser Vielfalt im katholischen Religionsunterricht. Eine explorative Videostudie
}

\author{
Ulrich Riegel (D) - Matthias Gronover • David Ambiel • \\ Malte Brügge-Feldhake • Sophia Jumpertz • Maximiliane Krämer • \\ Reinhold Boschki
}

Eingegangen: 12. März 2021 / Überarbeitet: 6. Juni 2021 / Angenommen: 5. Juli 2021 / Online publiziert: 25. November 2021

(C) Der/die Autor(en) 2021

Zusammenfassung In religionspädagogischen Theorien zum Religionsunterricht spielt religiöse Vielfalt eine zentrale Rolle. Empirische Studien zur Rolle und Bedeutung dieser Vielfalt im vorfindlichen Religionsunterricht stellen aber nach wie vor ein Desiderat religionsdidaktischer Forschung dar. Dieser Beitrag adressiert dieses Desiderat, indem er im Rahmen einer explorativen Videostudie untersucht, wie religiöse Vielfalt im katholischen Religionsunterricht thematisiert wird. Dazu greift er auf ein vierdimensionales Modell zurück, das religiöse Differenz entlang einer positionellen, einer stilistischen, einer kontextuellen und einer biographischen Dimension erfasst. Der Befund zeigt, dass vorfindlicher Unterricht religiöse Vielfalt

Prof. Dr. Ulrich Riegel $(\bowtie) \cdot$ Malte Brügge-Feldhake $\cdot$ Sophia Jumpertz

Seminar für Katholische Theologie, Universität Siegen, Adolf-Reichwein-Str. 2, 57068 Siegen,

Deutschland

E-Mail: Ulrich.Riegel@uni-siegen.de

Malte Brügge-Feldhake

E-Mail: malte.bfeldhake@uni-siegen.de

Sophia Jumpertz

E-Mail: sophia.jumpertz@student.uni-siegen.de

Prof. Dr. Matthias Gronover · David Ambiel · Maximiliane Krämer · Prof. Dr. Reinhold Boschki Fakultät für Katholische Theologie, Eberhard Karls Universität Tübingen,

Liebermeisterstr. 12, 72076 Tübingen, Deutschland

Prof. Dr. Matthias Gronover

E-Mail: matthias.gronover@uni-tuebingen.de

David Ambiel

E-Mail: david-andreas.ambiel@uni-tuebingen.de

Maximiliane Krämer

E-Mail: maximiliane-marie.kraemer@student.uni-tuebingen.de

Prof. Dr. Reinhold Boschki

E-Mail: reinhold.boschki@uni-tuebingen.de 
vor allem unter positionellen Aspekten verhandelt, wobei stilistische Aspekte eine Nebenrolle spielen. Dieser Befund wird abschließend diskutiert.

Schlüsselwörter Heterogenität · Religionsunterricht · Videostudie · Pluralitätsfähigkeit

\title{
Addressing religious heterogeneity in catholic religious education. An explorative video study
}

\begin{abstract}
Religious diversity plays a central role in theories of religious education. However, empirical studies on the role and significance of this diversity in teaching religious education still represent a desideratum of research. This article addresses this desideratum by examining how religious diversity is addressed in Catholic religious education within the framework of an explorative video study. To do so, it draws on a four-dimensional model that captures religious heterogeneity along a positional, a stylistic, a contextual, and a biographical dimension. The findings show that the teaching found negotiates religious diversity primarily under positional aspects, with stylistic aspects playing a secondary role. This finding is discussed in conclusion.
\end{abstract}

Keywords Heterogeneity · Religious Education · Video Study · Dealing with religious diversity

\section{Einleitung}

In der Mehrheit der Fälle wird der Religionsunterricht an deutschen Schulen konfessionell erteilt. Zwar drückt sich diese Konfessionalität nicht mehr in den Zielsetzungen des Faches aus, spielt aber eine Rolle für seinen Ausgangspunkt und seinen Bezugsrahmen (vgl. Englert 2015). Gleichzeitig findet der Religionsunterricht in einem sozialen Umfeld statt, das in weltanschaulicher und religiöser Hinsicht heterogen ist. Diese Vielfalt schreibt sich in den Religionsunterricht ein. Zum einen erweisen sich die Lerngruppen dieses Fachs nur noch in der Minderheit der Fälle als konfessionell bzw. religiös homogen, und selbst dann trifft man bei den Schülerinnen und Schülern auf unterschiedliche Einstellungen und Zugänge zu dieser Konfession bzw. Religion. Zum anderen muss der Religionsunterricht seine Themen vor dem Horizont besagter Vielfalt verhandeln, wenn er nicht als weltfremd und lebensfern erscheinen will. In der Konsequenz sucht der gegenwärtige konfessionelle Religionsunterricht seine Schülerinnen und Schüler zu einem kompetenten Umgang mit religiöser Vielfalt zu befähigen (vgl. Aslan 2017; Die Deutschen Bischöfe 2016; Rat der Evangelischen Kirche in Deutschland 2014).

Eine notwendige Voraussetzung, um diese Fähigkeit bei den Schülerinnen und Schülern zu erreichen, ist, dass religiöse Vielfalt im Religionsunterricht thematisiert wird. Wenn in diesem Fach die Mannigfaltigkeit religiöser und weltanschaulicher Überzeugungen und religiöser Vollzüge nicht angesprochen wird, kann auch keine Auseinandersetzung mit ihr stattfinden. Dabei spielt es erst einmal keine Rolle, ob 
religiöse Vielfalt planmäßig in die Auseinandersetzung im Unterricht eingespielt wird, z.B. durch ein entsprechendes Thema, oder aber spontan aus dem Unterrichtsprozess heraus erwächst, indem z.B. eine Schülerin eine in religiöser Hinsicht alternative Sichtweise der verhandelten Thematik einbringt. Es geht im Kern um die Frage, ob religiöse Vielfalt im konfessionellen Religionsunterricht auf der Ebene des Unterrichtsprozesses eine Rolle spielt und, wenn ja, auf welche Art und Weise sie im Bildungsprozess zum Tragen kommt. Auf diese Fragen liefert dieser Beitrag erste empirische Erkenntnisse. Dazu werden zuerst das begriffliche Umfeld dargestellt, innerhalb dessen die Religionspädagogik religiöse Vielfalt diskutiert (2), und die empirisch-religionspädagogische Forschungslage zur religiösen Vielfalt skizziert (3), um dann die Methode (4) und die empirischen Befunde (5) der vorliegenden Studie zu beschreiben. Eine Diskussion dieser Befunde beschließt den Beitrag (6).

\section{Das begriffliche Umfeld der religionspädagogischen Diskussion religiöser Vielfalt}

Seit den 1960er-Jahren wird das Phänomen der religiösen Vielfalt in der Religionspädagogik unter dem Begriff der Pluralität diskutiert, wobei es zuerst um den Kontext religiösen Lehrens und Lernens an der Schule ging, bevor Pluralität um die Jahrtausendwende zu einem religionspädagogischen „Konzeptbegriff mit programmatischem Anspruch“ (Simojoki 2020, S. 108) avanciert. So spricht sich Friedrich Schweitzer im programmatischen Band „Entwurf einer pluralitätsfähigen Religionspädagogik“ (Schweitzer et al. 2002) für einen starken Begriff von Pluralismus aus, der Pluralität als ein im Sinn des gesellschaftlichen Zusammenhalts zu bearbeitendes Phänomen begreift (vgl. Schweitzer 2002), und Rudolf Englert skizziert die Eckpunkte einer entsprechenden religionspädagogischen Programmatik (vgl. Englert 2002b). Ein pluralitätsfähiger Religionsunterricht habe demnach seiner in weltanschaulicher und religiöser Hinsicht vielgestaltigen Umwelt gerecht zu werden, indem die Schülerinnen und Schüler in diesem Fach befähigt werden, für sich selbst eine religiöse Orientierung zu gewinnen und respektvoll mit Menschen unterschiedlichster weltanschaulicher Standpunkte umzugehen.

In diesem Zusammenhang legte Rudolf Englert eine weitreichende theoretische Klärung des Begriffs religiöser Pluralität vor, indem er zwischen positionellen, stilistischen Differenzen einerseits und kontextuellen und biographischen Differenzen andererseits unterscheidet (vgl. Englert 2002a). Positionelle Differenzen beziehen sich auf die weltanschauliche Tradition der bzw. des Einzelnen oder einer Gemeinschaft. Dahingehend lassen sich nicht-religiöse und religiöse Sinnsysteme unterscheiden, wobei es bei Letzteren unterschiedliche Religionen (inter-religiöse Differenz) und innerhalb der einzelnen Religionen unterschiedliche Konfessionen oder Schulen (intra-religiöse bzw. inter-konfessionelle Differenz) gibt und auch innerhalb dieser Konfessionen und Schulen nochmals unterschiedliche Positionen eingenommen werden können (intra-konfessionelle Differenz). Stilistische Differenzen erfassen dagegen die Art und Weise, wie Religiosität ausgedrückt wird. Englert unterscheidet hier zwischen einem exklusiven Typ, der nur die Wahrheitsansprüche und Vollzüge der eigenen religiösen Tradition anerkennt, einem individualisierten Typ, der sich gemäß 
persönlicher Passung in unterschiedlichen religiösen Traditionen bedient, und einem säkularen Typ, der wenig bis nichts mit Religion anfangen kann. Positionelle und stilistische Differenzen werden durch kontextuelle und biographische Differenzen als „,intermittierende Faktoren“ (Englert 2002a, S. 19) bedingt. Kontextuelle Differenzen betreffen kohortenspezifische Unterschiede in der Religiosität, seien sie durch die typischen Sozialisationsbedingungen eines Geburtsjahrgangs, des Geschlechts oder des sozialen Milieus geprägt. Es geht hierbei um den Einfluss der generellen Lebenslage eines Menschen auf seine Religiosität. Biographische Differenzen beziehen sich auf den Einfluss der spezifischen Lebenslagen in der Vergangenheit eines konkreten Menschen auf seine aktuelle Religiosität. Sie erfassen somit die individuelle Verarbeitung von Erlebnissen, die für die eigene Religiosität von Bedeutung sind.

Englerts Begriffsbestimmung für die religionspädagogische Analyse religiöser Vielfalt erweist sich in mindestens doppelter Hinsicht als weiterführend. Erstens verweist sie darauf, dass es unterschiedliche Dimensionen dieser Vielfalt gibt und abhängig von der eingenommenen Dimension unterschiedliche Facetten des religiösen Spektrums erfasst werden. So mögen ein frommer Katholik und ein praktizierender Muslim in ihrer Haltung gegenüber einer zeitgenössischen Sexualmoral übereinstimmen (Stil), auch wenn sie zwei unterschiedlichen Religionen angehören (Position). Zweitens setzt er die vier Dimensionen religiöser Vielfalt in ein theoretisches Verhältnis zueinander. Definieren Position und Stil den Raum religiöser Orientierungsmöglichkeiten, legen Kontext und Biographie die Bandbreite dieser Orientierungsmöglichkeiten fest. Religiöse Vielfalt wird im Kontext des Pluralitätsbegriffs somit vor allem unter positionellen und stilistischen Perspektiven betrachtet, deren Ausprägungen sich u. a. durch kontextuelle und biographische Konstellationen erklären lassen.

In jüngerer Zeit wurde vorgeschlagen, den Pluralitätsbegriff durch den der Heterogenität abzulösen (vgl. Grümme 2017). Der Mehrwert des neuen Begriffs wird neben seiner pädagogischen Anschlussfähigkeit (z. B. Trautmann und Wischer 2011) vor allem in seiner Offenheit gegenüber verschiedenen Dimensionen und Ausprägungen von Differenz gesehen. Adressiert der Pluralitätsbegriff vor allem weltanschauliche Vielfalt, beinhaltet der Heterogenitätsbegriff auch Vielfalt in körperlicher, geschlechtlicher, sozialer, ethnischer, intellektueller, usw. Hinsicht, wodurch weitergehende religionsbezogene Differenzen sichtbar werden (Grümme 2021). Zudem werfe der Heterogenitätsbegriff die Frage nach Machverhältnissen auf, weshalb er eng mit dem allgemeinen Ziel nach Bildungsgerechtigkeit verzahnt sei (vgl. Grümme 2014). Gegenwärtig wird der Heterogenitätsbegriff religionspädagogisch vor allem in doppelter Hinsicht verwendet. Zum einen wird er als analytischer Begriff herangezogen, um die vielfältigen Differenzen, die religionspädagogisches Tun prägen, zu diskutieren (z.B. Büttner et al. 2018; Gronover 2012; Reis 2021). Zum anderen wird er als normativer Begriff unter dem Titel einer ,,aufgeklärten Heterogenität“ zum Maßstab religionspädagogischer Konzeptbildung ausgerufen (Grümme 2018).

Mit Blick auf religiöse Vielfalt führt der Heterogenitätsbegriff somit nicht zu neuen Kategorien, weitet aber den Fokus in mindestens doppelter Hinsicht. Erstens führt er neben der Beachtung von Unterschieden auch zur Berücksichtigung von Ungleichheiten (Grümme 2017, S. 17). Einer heterogenitätssensiblen Religionspäd- 
agogik geht es bei der Analyse von religiöser Vielfalt auch darum, wie sich Unterschiede und Ungleichheiten ,gegenseitig beeinflussen, vorantreiben, dynamisieren oder auch relativieren“ (Grümme 2017, S. 51). Der Heterogenitätsbegriff schreibt der Diskussion religiöser Vielfalt somit eine normative Perspektive ein. Zweitens rücken im Rahmen des Heterogenitätsbegriffs die Kontexte religiöser Vielfalt stärker in den Mittelpunkt. Die bei Englert als intermittierende Faktoren bestimmten Dimensionen des Kontextes und der Biographie werden als entscheidende Analysekategorien für die Lernvoraussetzungen und -prozesse des Religionsunterrichts herangezogen (Domsgen und Witten 2018). Ferner avancieren beide Dimensionen unter der Prämisse, dass Heterogenität ,,als zentrale Kategorie einer zeitgemäßen religiösen Bildung“ (Gärtner 2015, S. 14) aufgefasst wird, unter den Begriffen des milieusensiblen und biographischen Lernens zu entscheidenden Bezugspunkten des Unterrichtsgeschehens im Fach Religion (Gärtner 2015, S. 114 ff.).

Vergleicht man die beiden religionspädagogischen Diskurse zur religiösen Vielfalt, setzen sowohl der Pluralitäts- wie auch der Heterogenitätsbegriff bei analogen Phänomenen an, diskutieren sie jedoch unter unterschiedlichen theoretischen Konnotationen (Schweitzer 2021). Beide gründen in der Einsicht, dass Kinder und Jugendliche angesichts einer immer stärker werdenden Vielfalt aufwachsen. Allerdings verdankt sich der Pluralitätsbegriff einem religionsbezogenen Diskurs und nimmt somit religiöse Vielfalt als zentrales Phänomen in den Blick, während der Heterogenitätsbegriff dem pädagogischen Ringen um einen angemessenen Umgang mit Vielfalt an sich entstammt, wodurch er verschiedene Aspekte von Vielfalt thematisiert und religiöse Vielfalt - wenn überhaupt - als einen Aspekt unter anderen von Vielfältigkeit berücksichtigt. Für die religionspädagogische Diskussion stellt der Heterogenitätsbegriff damit gegenüber dem Pluralitätsbegriff einerseits eine Perspektivenerweiterung dar. Exemplarisch wird dieser Unterschied an der Rolle deutlich, die der Kontext und die Biographie in beiden Begrifflichkeiten spielen. Gelten sie im Pluralitätsbegriff als bedingende Faktoren, stellen sie im Heterogenitätsbegriff zentrale Aspekte von Vielfalt dar. Andererseits verliert das Konzept religiöser Vielfalt innerhalb des Heterogenitätsbegriffs seine konzeptuelle Schärfe, weil biographische und kontextuelle Aspekte keine genuinen Kategorien des religionsbezogenen Diskurses sind. Nach Friedrich Schweitzer scheint der Pluralitätsbegriff geeignet zu sein, die Innenperspektive auf religiöse Vielfalt stark zu machen, während der Heterogenitätsbegriff eher eine Außenperspektive auf diese Vielfalt anbietet (Schweitzer 2021, S. 34 f.).

\section{Empirisch-religionspädagogische Forschung zu religiöser Vielfalt}

Die vorliegende empirische Forschung innerhalb der Religionspädagogik zu religiöser Vielfalt orientiert sich auch in jüngerer Zeit vor allem am Pluralitätsbegriff. So liegen vergleichsweise viele Studien zur Rolle individueller Religiosität von Kindern und Jugendlichen als Kontextfaktor des Religionsunterrichts vor, die sich auf positionelle (z. B. Schweitzer et al. 2018; Sorg 2020; Ziebertz et al. 2003) und stilistische Aspekte von Religiosität beziehen (z. B. Könemann 2002; Ulfat 2017). Kontextuelle Perspektiven werden nur vereinzelt aufgegriffen (v.a. zur Rolle von Gender: vgl. 
Arzt 1999; Riegel 2004), ebenso biographische (z. B. Kaupp 2005; Reese-Schnitker 2006). Damit stehen die beiden Dimensionen im Fokus dieses Forschungsstrangs, die beim Pluralitätsbegriff die entscheidende Rolle spielen. Inhaltlich legen es diese Studien nahe, dass Lerngruppen im heutigen Religionsunterricht vielfältig sind, wobei vor allem die große Bandbreite individueller Religiosität innerhalb der verschiedenen christlichen Konfessionen deutlich wird.

Ein zweiter Forschungsstrang zu religiöser Vielfalt untersucht deren Bedeutung im professionellen Selbstverständnis von Religionslehrpersonen (vgl. Überblick bei Riegel 2021, S. 52 ff.). Diese Studien zeigen auf, dass Lehrpersonen religiöse Vielfalt vor allem als positionelle Differenz wahrnehmen, denn sie wird von diesen in der Regel über religiöse Zugehörigkeit operationalisiert. So wird beim Stichwort religiöse Vielfalt nahezu durchgehend angeführt, dass in den Lerngruppen des konfessionellen Religionsunterrichts auch nicht-christliche Schülerinnen und Schüler sitzen, sowie solche der Geschwisterkonfession. Unterschiede innerhalb der Kinder und Jugendlichen der eigenen Konfession werden dagegen kaum zur Sprache gebracht. Auch bei der Einschätzung der Lernchancen religiöser Vielfalt im Religionsunterricht wird in der Regel überlegt, welche Anknüpfungspunkte nicht-christliche und säkulare Schülerinnen und Schüler bieten. Außerdem trifft man bei sehr vielen Kolleginnen und Kollegen auf eine gewisse Sympathie für konfessionell-kooperative Formate des Religionsunterrichts, in dennen evangelische und katholische Schülerinnen und Schüler gemeinsam lernen (vgl. Gronover et al. 2021). Wiederum sind es die Kategorien, die den Pluralitätsbegriff prägen, die die Wahrnehmung religiöser Vielfalt von Religionslehrpersonen primär leiten. Inhaltlich verweisen diese Studien darauf, dass besagte Lehrkräfte sich als didaktisch gut auf die religiöse Vielfalt ihrer Lerngruppen eingestellt erachten und in ihrem Unterricht sensibel mit ihr umgehen wollen. Allerdings erbrachte eine aktuelle Analyse von elf Expert*inneninterviews zum Umgang mit religiöser Vielfalt im interreligiösen Lernen vor allem emotional distanzierte Haltungen, gemäß derer der interreligiöse Dialog zwar aus gesellschaftspolitischen Gründen angestrebt wird, es jedoch kaum Bereitschaft gibt, sich selbst durch diesen Dialog verändern zu lassen (vgl. Mauritz et al. 2020). Gleichzeitig dominierte in den subjektiven Theorien dieses Samples zum interreligiösen Lernen die Instruktion deutlich gegenüber der Begegnung oder der Positionierung als Lernform (vgl. Reis et al. 2020).

Zur Thematisierung religiöser Vielfalt im Unterrichtsgeschehen selbst gibt es bislang dagegen nur wenige Studien, die noch dazu sehr spezifische Fragestellung aufweisen. Eine explorative Untersuchung von 15 Unterrichtseinheiten aus NordrheinWestfalen mit insgesamt 116 Unterrichtsstunden zur Thematisierung von Religion im katholischen Religionsunterricht ergab, dass die Lehrpersonen vor allem den katholischen Blick auf die Sachverhalte zur Geltung brachten (vgl. Riegel und Delling 2019). Lebensweltliche Bezüge oder die Perspektive anderer Religionen wurden vor allem funktional eingestreut, um die katholische Perspektive klarer herauszustellen. Nur in zwei der 15 Einheiten wurden nicht-christliche Inhalte in ihrem Eigenwert thematisiert. Ferner fragte Barbara Asbrand aus einem erziehungswissenschaftlichen Erkenntnisinteresse heraus, wie sich religiöse Vielfalt innerhalb einer Lerngruppe des Religionsunterrichts auf die unterrichtlichen Interaktionen auswirkt (Asbrand 2000; auch: Buchardt 2010). Sie konnte zeigen, dass im Unterrichtsgeschehen viel- 
fache - oft unterbewusst verlaufende - Prozesse von unangemessener Fremdzuschreibung und Ausgrenzung über die Religionszugehörigkeit der Schülerinnen und Schüler erfolgen. Schließlich neigen die Lehrpersonen einer Essener Studie dazu, nahezu ausschließlich über Religion zu informieren, was dazu führe, dass die Schülerinnen und Schüler religiöse Vielfalt zwar kennen lernten, diese jedoch nicht im Sinne eines kompetenten Umgangs mit dieser Vielfalt bearbeiten könnten, weil es kaum zu einer Auseinandersetzung mit religiösen Geltungsansprüchen komme (vgl. Englert et al. 2014). In allen diesen Studien wurde religiöse Vielfalt im Kontext des Pluralitätsbegriffs operationalisiert. Berücksichtigt man zusätzlich empirische Studien zum interreligiösen Lernen, die religiöse Vielfalt per Definition positionell oder stilistisch thematisieren, gelingt es diesen Ansätzen, Wissen über andere Religionen zu vermitteln, während die Befunde zur Befähigung zur Perspektivübernahmen ambivalent ausfallen und der Effekt interreligiösen Lernens auf die Einstellungen der Schülerinnen und Schüler anderen Religionen gegenüber marginal bleibt (Unser 2018).

Auch wenn alle referierten Studien in der Regel regional beschränkt und schulartspezifisch und damit nicht repräsentativ angelegt sind, konvergieren sie doch im Eindruck, dass heutige Religionslehrpersonen der in ihrer Umwelt und in ihren Lerngruppen vorfindlichen religiösen Vielfalt mehrheitlich positiv gegenüberstehen. Wie sich diese Einstellung im Unterrichtsgeschehen auswirkt, stellt jedoch ein Desiderat religionspädagogischer Forschung dar. Wird religiöse Vielfalt im vorfindlichen (konfessionellen) Religionsunterricht angesprochen und falls ja, eher unter den Prämissen des Pluralitätsbegriffs oder denen des Heterogenitätsbegriffs? Auf dieses Desiderat reagiert der vorliegende Beitrag, in dem er die Frage nach der Thematisierung religiöser Vielfalt im katholischen Religionsunterricht stellt. Er richtet seinen Fokus damit auf den Inhaltsaspekt der unterrichtlichen Auseinandersetzung. Eine derartige Festlegung ist auf der einen Seite willkürlich, denn religiöse Vielfalt im Unterrichtsgeschehen wird durch vielfältige Faktoren bedingt. Auf der anderen Seite liegt eine derartige Fokussierung der Fragestellung aus forschungsökonomischen Gründen nahe. Auf keinen Fall ist mit dieser Entscheidung impliziert, dass andere Fokussierungen, etwa auf die Unterrichtsbeteiligung der Schülerinnen und Schüler in Abhängigkeit zu ihrer konfessionellen bzw. religiösen Zugehörigkeit, weniger wichtig wären. Die generelle Forschungsfrage dieses Beitrags lautet deshalb: Wie wird religiöse Vielfalt im katholischen Religionsunterricht auf der Inhaltsebene thematisiert?

\section{Methode}

Da der Fokus dieses Beitrags auf dem Geschehen im Unterricht liegt, wird die Videoanalyse als empirischer Zugriff zum Feld gewählt (vgl. Riegel 2018). Unterrichtsvideos ermöglichen die differenzierte Rekonstruktion von Unterrichtsprozessen, wobei optische und akustische Informationen zur Verfügung stehen. Die Aufnahme erfolgte über eine Lehrerkamera im rückwärtigen Teil des Klassenraums und eine Schülerkamera, die in einer vorderen Ecke des Raums platziert war, sowie zwei eigenständige Hochleistungsmikrophone (vgl. Seidel et al. 2005). In der Kombina- 
tion beider Videos und beider Tonspuren kann der Unterrichtsprozess, so lange er im Plenum abläuft, präzise nachgezeichnet werden. Lediglich Einzel- und Gruppenarbeiten lassen sich durch dieses Aufnahmesetting nur bedingt erfassen. Allerdings wurden Handkameras, die auch diese Arbeitsprozesse dokumentieren könnten, von den angefragten Lehrpersonen als zu invasiv erachtet und deshalb nicht eingesetzt.

Insgesamt wurden 42 Stunden per Video dokumentiert. Ihre Aufzeichnung erfolgte im Rahmen des DFG-Projekts ,Umgang mit religiöser Heterogenität im konfessionellen Religionsunterricht“. Für dieses Projekt konnten 23 Lehrpersonen aus Baden-Württemberg und Nordrhein-Westfalen gewonnen werden. Sie waren eingeladen, zwei Stunden aus unterschiedlichen Unterrichtsreihen videographieren zu lassen, wobei sie um den Zweck der Untersuchung wussten und gebeten wurden, nach Möglichkeit je eine Stunde zum interreligiösen und eine zum ethischen Lernen anzubieten. Die beiden thematischen Schwerpunkte wurden ausgewählt, weil sie in besonderer Weise geeignet erscheinen, religiöse und weltanschauliche Vielfalt hervorzubringen. Allerdings konnten die angefragten Lehrpersonen diesem Wunsch nicht immer nachkommen, weil das schulinterne Curriculum andere Themen vorsah. Letztlich behandeln 59\% der aufgezeichneten Stunden ein ethisches Thema und $18 \%$ ein interreligiöses. Die restlichen $23 \%$ beziehen sich auf ein religiöses Thema. Ferner konnte in vier Fällen die zweite Videoaufzeichnung aus gesundheitlichen oder schulorganisatorischen Gründen nicht stattfinden. In der Folge besteht die Stichprobe dieses Beitrags aus $N=42$ Stunden. Von diesen fanden 13 an weiterführenden allgemeinbildenden Schulen statt und 29 an beruflichen Schulen. Die Länge der Stunden variiert zwischen 37 und $91 \mathrm{~min}$.

Die Analyse der Unterrichtsvideos geschah via hoch inferentem Rating (vgl. Lotz et al. 2013). Das einschlägige Ratingmanual setzt bei der oben beschriebenen Begriffsklärung religiöser Vielfalt Rudolf Englerts an, indem es die vier Perspektiven Position, Stil, Kontext und Biographie unterscheidet (vgl. Englert 2002a). Dabei bezieht sich der positionelle Aspekt auf die Gegenstandsdimension von Religion. Er zeigt sich in im Unterricht geäußerten oder angesprochenen Sachverhalten, Theorien, Glaubenssätzen, Einstellungen und Überzeugungen, insofern sie etablierten religiösen Traditionen (z.B. Christentum, Islam, etc.) oder säkularen Sinnmustern (Humanismus, Ökosophie, etc.) zugeordnet werden können. Der stilistische Aspekt steht für die Ausdrucksformen, wie Religion bzw. säkulare Sinnmuster individuell oder kollektiv gelebt und ausgeübt werden. Er kann als Unterrichtsgegenstand ebenso angetroffen werden wie auch in der Art und Weise, wie im Unterricht über Religion gesprochen wird. Beim kontextuellen Aspekt geht es um Prägungen von Religion, die durch das soziale Umfeld, das Geschlecht oder die Geburtskohorte bedingt sind. Er kann sich in der Aussage oder im Verhalten einer Person im Unterricht zeigen, evtl. auch durch das Unterrichtsmaterial, das im Video sichtbar ist, eingespielt werden, muss aber stets auf eine über-individuelle Lebenslage rückführbar sein, die sich in der betreffenden Person exemplarisch ausdrückt. Der biographische Aspekt erfasst schließlich die Art und Weise, wie ein Individuum seine eigene Vergangenheit religionsbezogen verarbeitet und zu einem religionsbezogenen Lebensgefühl verdichtet. Für diesen Aspekt muss die Erfahrung selbst, um die es geht, nicht notwendig etwas mit Religion zu tun haben. Sie muss jedoch in der verhandelten Situation auf Religion bezogen werden. 
Um diese vier Perspektiven empirisch handhabbar zu operationalisieren, war es notwendig, in einem ersten Schritt nach dem Vorliegen entsprechender Aspekte zu fragen, um anhand dieser Aspekte in einem zweiten Schritt abzuleiten, ob auch eine Differenz vorliegt. Das Ratingmanual enthält somit für jede der vier Dimensionen zwei Ratingvorschriften. Für das Rating im ersten Schritt ist entscheidend, welche Rolle jeder der vier Aspekte in einer Analyseeinheit für den beobachtbaren Unterrichtsprozess spielt. Fünf Ratingkategorien sind möglich. Eine 4 wird vergeben, falls der jeweilige Aspekt eine zentrale Rolle spielt, d.h. den thematischen Kern der Sequenz prägt und/oder ein dominantes Element in den Schülergesprächen ist. Eine 3 repräsentiert eine deutlich erkennbare, aber nicht zentrale Rolle des betreffenden Aspekts, d.h. er prägt Teile der Sequenz und/oder ist ein wichtiges Element einiger Schülergespräche. Eine 2 steht für eine untergeordnete Rolle des zu ratenden Aspekts, insofern er in der Analysesequenz angetroffen wird, ohne sie zu prägen. Die 1 wird eingetragen, wenn der Aspekt in der Sequenz keine Rolle spielt. Schließlich kennzeichnet eine 99 die Analyseeinheiten, die nicht im Sinn des jeweiligen Aspekts deutbar waren. Das ist z. B. häufig in den Unterrichtsphasen der Fall, in denen in Gruppen gearbeitet wird. Eine Analyseeinheit erstreckt sich dabei auf eine Minute. Diese Zeitspanne ist zum einen lange genug, um einen thematischen $\mathrm{Zu}$ sammenhang rekonstruieren zu können, zum anderen aber auch wieder kurz genug, um eine differenzierte Analyse der thematischen Dynamik der Unterrichtsprozesse zu ermöglichen. Die Stichprobe für den ersten Analyseschritt besteht damit aus den $N_{t s}=2220 \mathrm{~min}$ der aufgezeichneten Unterrichtsstunden.

Im zweiten Schritt wurde dann im Anschluss an jede Stunde ermessen, inwiefern sich in den erfassten Aspekten jeder Dimension eine Differenz ausdrückt. Erst mit dieser Analyse wird somit attestiert, ob religiöse Vielfalt in der Unterrichtsstunde eine Rolle spielt. Eine Differenz liegt dann vor, wenn sich mindestens zwei unterscheidbare Positionen oder Stile in der thematischen Struktur der Stunde unterscheiden lassen oder aber mindestens zwei kontextuelle oder biographische Ausprägungen angetroffen werden können. Ist dies nicht der Fall, wird die gesamte Stunde hinsichtlich des betreffenden Aspekts mit 1 geratet. Wird eine Differenz angetroffen, wird entschieden, ob sie im thematischen Verlauf der Stunde eine untergeordnete (2), eine deutlich erkennbare (3) oder eine zentrale Rolle (4) gespielt hat. Die Analyseeinheit im zweiten Schritt bezieht sich damit auf die gesamte Stunde. Die Stichprobe für den zweiten Analyseschritt besteht damit aus den $N_{e s}=42$ aufgezeichneten Unterrichtsstunden.

Anhand der theoretischen Präzisierungen des Vielfaltsbegriffs in der Operationalisierung beider Analyseschritte lässt sich die Forschungsfrage dieses Beitrags differenzierter formulieren:

- F1: Welche Bedeutung haben positionelle, stilistische, kontextuelle und biographische Aspekte für die Inhaltsebene im katholischen Religionsunterricht?

- F2: Welche Bedeutung haben positionelle, stilistische, kontextuelle und biographische Differenzen auf der Inhaltsebene im katholischen Religionsunterricht?

Außerdem erlaubt es die Bestimmung der Rolle religiöser Differenzen entlang von vier Dimensionen, die konkrete Konstellation zu bestimmen, gemäß der religiöse Vielfalt innerhalb einer Stunde thematisiert wird. Diese Konstellation wird dabei 
definiert als Kombination der Differenzdimensionen innerhalb einer Religionsstunde, die mindestens mit 3 (= erkennbar) geratet wurden. Letzteres ist notwendig, um sachlich angemessen von einer Thematisierung der jeweiligen Differenzdimension sprechen zu können. Die entsprechend Forschungsfrage lautet:

- F3: In welchen Konstellationen finden sich positionelle, stilistische, kontextuelle und biographische Differenzen innerhalb der Religionsstunden, sofern sie mindestens mit 3 geratet wurden?

Zur Beantwortung der ersten beiden Forschungsfragen werden Häufigkeitsauszählungen herangezogen, bei der ersten Forschungsfrage wird zusätzlich der Median berechnet. Für die Beantwortung der dritten Forschungsfrage werden aus den Ratings zu den vier Differenzdimensionen Quadrupel gebildet, die daraufhin analysiert werden, wie oft sich eine 3 oder eine 4 darin findet und in welchen Kombinationen man auf diese Zahlenwerte trifft.

Das Rating der aufgezeichneten Unterrichtsstunden wurde von zwei Raterinnen vollzogen. Sie wurden zuerst anhand des Codebuchs und einer Beispielstunde, die nicht zum Datensatz dieses Beitrags gehört, geschult. Dann wurde eine Stunde des Datensatzes unabhängig voneinander geratet, die Inter-Rater-Reliabilität (CohensKappa) berechnet und abweichende Ratings besprochen. Dieses Verfahren wurde so lange wiederholt, bis für Cohens-Kappa in zwei aufeinanderfolgenden Ratings in allen acht Dimensionen galt: $\kappa>0,70$ (vgl. Wirtz und Caspar 2002, S. 59). Dieses Ziel wurde bei den Videos neun und zehn erreicht, sodass die restlichen Videos unter beiden Raterinnen aufgeteilt werden konnten.

\section{Ergebnisse}

Die erste Forschungsfrage adressiert die Thematisierung religiös relevanter Aspekte im Unterrichtsprozess (vgl. Tab. 1).

Die Verteilung der Relevanz-Kategorien innerhalb der vier Dimensionen, entlang derer Religion und Religiosität angesprochen werden können, ergibt eine deutliche Dominanz positioneller Aspekte. In 58,3\% aller untersuchten Unterrichtssegmente

Tab. 1 Bedeutung des positionellen, stilistischen, kontextuellen und biographischen Aspekts auf der Inhaltsebene des katholischen Religionsunterrichts

\begin{tabular}{|c|c|c|c|c|c|c|c|c|}
\hline \multirow[b]{2}{*}{ Relevanz } & \multicolumn{2}{|c|}{$\begin{array}{l}\text { Positioneller } \\
\text { Aspekt }\end{array}$} & \multicolumn{2}{|c|}{$\begin{array}{l}\text { Stilistischer } \\
\text { Aspekt }\end{array}$} & \multicolumn{2}{|c|}{$\begin{array}{l}\text { Kontextueller } \\
\text { Aspekt }\end{array}$} & \multicolumn{2}{|c|}{$\begin{array}{l}\text { Biographischer } \\
\text { Aspekt }\end{array}$} \\
\hline & $N$ & $\%$ & $N$ & $\%$ & $N$ & $\%$ & $N$ & $\%$ \\
\hline Keine & 152 & 6,8 & 1019 & 45,9 & 1675 & 75,5 & 1623 & 73,1 \\
\hline Niedrige & 293 & 13,2 & 407 & 18,3 & 46 & 2,1 & 71 & 3,2 \\
\hline Größere & 404 & 18,2 & 203 & 9,1 & 15 & 0,7 & 26 & 1,2 \\
\hline Große & 890 & 40,1 & 112 & 5,0 & 5 & 0,5 & 21 & 0,9 \\
\hline $\begin{array}{l}\text { Nicht zu- } \\
\text { ordenbar }\end{array}$ & 479 & 21,6 & 479 & 21,6 & 479 & 21,6 & 479 & 21,6 \\
\hline Gesamt & 2220 & 100 & 2220 & 100 & 2220 & 100 & 2220 & 100 \\
\hline
\end{tabular}

Analyseeinheit ist eine Minute im Religionsunterricht 
Tab. 2 Rolle positioneller, stilistischer, kontextueller und biographischer Differenz auf der Inhaltsebene des katholischen Religionsunterrichts

\begin{tabular}{|c|c|c|c|c|c|c|c|c|}
\hline \multirow[b]{2}{*}{ Rolle } & \multicolumn{2}{|c|}{$\begin{array}{l}\text { Positionelle Diffe- } \\
\text { renz }\end{array}$} & \multicolumn{2}{|c|}{$\begin{array}{l}\text { Stilistische Diffe- } \\
\text { renz }\end{array}$} & \multicolumn{2}{|c|}{$\begin{array}{l}\text { Kontextuelle Diffe- } \\
\text { renz }\end{array}$} & \multicolumn{2}{|c|}{$\begin{array}{l}\text { Biographische } \\
\text { Differenz }\end{array}$} \\
\hline & $N$ & $\%$ & $N$ & $\%$ & $N$ & $\%$ & $N$ & $\%$ \\
\hline Keine & 1 & 2,4 & 9 & 21,4 & 36 & 85,7 & 33 & 78,6 \\
\hline $\begin{array}{l}\text { Unter- } \\
\text { geordnet }\end{array}$ & 5 & 11,9 & 15 & 35,7 & 5 & 11,9 & 9 & 21,4 \\
\hline Erkennbar & 10 & 23,8 & 15 & 35,7 & 1 & 2,4 & 0 & 0,0 \\
\hline Zentral & 26 & 61,9 & 3 & 7,1 & 0 & 0,0 & 0 & 0,0 \\
\hline Gesamt & 42 & 100 & 42 & 100 & 42 & 100 & 42 & 100 \\
\hline
\end{tabular}

Analyseeinheit ist die gesamte Religionsstunde

spielt sie mindestens eine größere Rolle, in 40,1\% sogar eine große. Stilistische Aspekte von Religion und Religiosität werden in ca. 10\% der Unterrichtssegmente so angesprochen, dass man von einer mindestens größeren Rolle sprechen kann. Kontextuelle und biographische Aspekte stellen, wenn überhaupt, nur Nebenthemen im Unterrichtsprozess dar. Diese Dominanz inhaltlicher Positionalität schlägt sich auch im Median der vier Aspekte nieder. Berücksichtigt man nur die Unterrichtssegmente, in denen die Bedeutung der vier Aspekte bestimmbar war, gilt für den Median des positionellen Aspekts $M d n=4$, während für die anderen drei Aspekte $M d n=1$ gilt.

Forschungsfrage 2 bezieht sich auf die Differenzen, durch die sich religiöse Vielfalt innerhalb der vier Dimensionen von Religion ausdrückt. Religiöse Vielfalt liegt dann auf der Inhaltsebene einer Stunde vor, wenn mindestens zwei klar unterscheidbare Facetten entlang einer der vier Dimensionen identifiziert werden können. In diesen Fällen wurde dann bestimmt, welche Rolle religiöse Vielfalt für die Stunde gespielt hat (vgl. Tab. 2).

In positioneller Hinsicht findet sich nur eine Stunde, in der religiöse Vielfalt überhaupt nicht anzutreffen ist $(2,4 \%)$. In weiteren fünf Stunden spielt sie eine untergeordnete Rolle (11,9\%). In 26 Religionsstunden (61,9\%) erweisen sich dagegen mindestens zwei unterschiedliche positionelle Aspekte als zentraler Unterrichtsgegenstand. Ein vergleichbares Gewicht kommt bei den anderen Dimensionen von Religion nur der stilistischen Differenz zu, die in drei Fällen $(7,1 \%)$ eine zentrale Rolle auf der Inhaltsebene der Religionsstunde spielt. In weiteren 15 Religionsstunden $(35,7 \%)$ werden erkennbar stilistische Unterschiede verhandelt, ohne dass diese sich als zentral für die im Unterricht verhandelten Inhalte erweisen. Die beiden übrigen Dimensionen religiöser Vielfalt sind hinsichtlich ihrer Thematisierung im Religionsunterricht nahezu ohne Belang. In einem Fall $(2,4 \%)$ wirken sich kontextuelle Differenzen erkennbar auf die im Unterricht verhandelten Inhalte aus. Ansonsten finden sich noch fünf Stunden $(11,9 \%)$, in denen kontextuelle Differenzen eine untergeordnete Rolle spielen. In neun Stunden $(21,4 \%)$ gilt dies für biographische Differenzen.

Die dritte Forschungsfrage bezieht sich auf die Konstellation, gemäß der religiöse Vielfalt in den Religionsstunden thematisiert wird. Um für eine solche Konstellation berücksichtigt zu werden, muss eine Dimension mit 3 (= erkennbar) oder 4 (= zen- 
tral) geratet worden sein. In einer Stunde zum Islam trifft das auf drei Dimensionen $\mathrm{zu}$, nämlich auf die positionelle, die stilistische und die kontextuelle. In allen anderen Fällen prägen höchstens zwei Dimensionen die Thematisierung von Vielfalt auf der Inhaltsebene, wobei es sich hierbei immer um die positionelle und bzw. oder die stilistische Differenz handelt. In drei Stunden sind sowohl die positionelle als auch die stilistische Differenz zentral für das inhaltliche Geschehen. In weiteren 13 Stunden ist die positionelle Differenz zentral und die stilistische erkennbar. In einer Stunde schließlich sind sowohl die positionelle als auch die stilistische Differenz erkennbar. Für 17 Religionsstunden gilt, dass ausschließlich die positionelle Differenz entweder zentral oder erkennbar ausgeprägt ist. Damit finden sich auch sechs Stunden im Sample, in denen religiöse Vielfalt praktisch keine Rolle spielt, d.h. keine der vier Dimensionen mindestens mit 3 geratet wurde. Zusammenfassend bilden positionelle Differenzen den Fixpunkt in den beobachteten Konstellationen religiöser Vielfalt.

\section{Diskussion}

Dieser Beitrag untersucht, wie religiöse Vielfalt im (katholischen) Religionsunterricht thematisiert wird. Er richtet seinen Fokus damit auf die Inhaltsebene des Unterrichtsprozesses. Dabei orientiert er sich an einem vierdimensionalen, deskriptiven Modell religiöser Vielfalt, indem er zwischen positionellen, stilistischen, kontextuellen und biographischen Zugängen unterscheidet. In der hier zugrundeliegenden Studie wurden Unterrichtsvideographien zunächst auf positionelle, stilistische, kontextuelle und biographische Aspekte hin analysiert, um dann in einem zweiten Schritt aus den Aspekten entsprechende Differenzen abzuleiten. Eine derartige konzeptuelle Differenzierung der Inhaltsebene wird u.W. zum ersten Mal explizit in der religionsdidaktischen Unterrichtsforschung verwendet, insofern sich diese auf inhaltliche Aspekte des Unterrichtsprozesses bezieht. Vorliegende Studien mit einem vergleichbaren Erkenntnisinteresse sprechen in der Regel allgemein vom Inhalt des Unterrichts und nehmen vor allem das in den Blick, was in der vorliegenden Untersuchung als positioneller Aspekt verhandelt wird (z. B. Englert et al. 2014; Kumlehn 2018, S. 94 ff.; Riegel und Delling 2019; Wagensommer und Schweitzer 2012).

Die Antwort auf die erste Forschungsfrage zeigt, dass derartige positionelle Aspekte das inhaltliche Geschehen der untersuchten Stunden tatsächlich prägen. Religion wird hauptsächlich anhand von Sachverhalten, Glaubenssätzen, Einstellungen und Überzeugungen verhandelt. Die Art und Weise, wie derartige Sachverhalte, Glaubenssätze, Einstellungen und Überzeugungen gelebt werden, d.h. stilistische Aspekte, spielen dagegen eine untergeordnete Rolle. Kontextuelle und biographische Aspekte werden fast gar nicht angesprochen. Diese Beobachtung legt den Schluss nahe, dass religiöse und weltanschauliche Traditionen im Unterricht als Sache ,an sich" thematisiert werden, d.h. als objektiv rekonstruierbare Sachverhalte, die unabhängig von ihrer Rezeption in sozialer Praxis angemessen erfasst werden können. Dieser Zugang zu Religion wird in jüngerer Zeit unter dem Stichwort der ,Essentialisierung von Religion“ vor allem in den Diskursen zum interreligiösen Lernen (z. B. Meyer 2019; Sajak 2012) und zum religionskundlichen Zugriff auf Religion kritisch 
hinterfragt (z. B. Kilchsberger 2015; Owen 2011). In beiden Fällen wird angemerkt, dass Religion nicht nur ein theoretisches System aus Glaubenssätzen und -vollzügen sowie moralischen Normen, sondern auf gelebte Praxis angewiesen und in dieser vielgestaltig und kontingent sei.

Angesichts der Dominanz positioneller Aspekte im Religionsunterricht kann es nicht verwundern, wenn religiöse Vielfalt vor allem entlang dieser Dimension thematisiert wird (vgl. Forschungsfrage 2). Allerdings spielen stilistische Differenzen in 18 der 42 videographierten Stunden eine mindestens deutlich erkennbare Rolle. Dieser Anteil ist bemerkenswert hoch, wenn man bedenkt, dass stilistische Aspekte nur in 315 von 2220 untersuchten Minuten Religionsunterricht eine größere Rolle gespielt haben. Dieses Verhältnis legt den Schluss nahe, dass die Art und Weise, wie ein religiöses Bekenntnis oder eine weltanschauliche Haltung gelebt wird, insbesondere dann im Unterricht angesprochen wird, wenn es um religiöse Vielfalt geht. Denkbar ist aber auch der umgekehrte Schluss, dass immer dann, wenn der Unterricht von der Angebotsseite her die Möglichkeit eröffnet, über den Stil ins Gespräch zu kommen, religiöse Vielfalt in besonderer Weise offenbar wird. Die Stilistik scheint im Religionsunterricht damit ein für die Schülerinnen und Schüler gut rezipierbarer Indikator für religiöse Vielfalt zu sein. Ob es sich dabei um Differenzen auf einer generellen Ebene handelt (z.B. „der“ Moslem betet so, ,der“ Christ so) oder aber individuell spezifische Praktiken verhandelt werden, kann aufgrund unserer Analysemethode nicht bestimmt werden. Hierzu wäre ein qualitativer Blick in die Unterrichtssequenzen notwendig.

Für die Konstellationen, in denen religiöse Vielfalt im Religionsunterricht thematisiert wird (vgl. Forschungsfrage 3), bedeutet das, dass positionelle Differenz die Leitkategorie darstellt, die in vielen Fällen durch stilistische Differenzen ergänzt wird. Nur in einem Fall wird zusätzlich eine kontextuelle Differenz eingespielt. Hierbei handelt es sich um eine Stunde zum Image des Islam, in der herausgearbeitet wird, wie stark seine Assoziation mit Terrorismus durch die Wahrnehmung aktueller Ereignisse und deren mediale Darstellung bedingt ist.

Bezieht man diesen Befund auf den religionspädagogischen Diskurs religiöser Vielfalt, scheint die Thematisierung dieser Vielfalt auch im vorfindlichen Religionsunterricht vor allem am Pluralitätsbegriff orientiert zu sein, während der spezifische Fokus des Heterogenitätsbegriffs praktisch keine Rolle spielt. Letzteres liegt einerseits nahe, weil der Heterogenitätsbegriff noch relativ jung ist in der religionspädagogischen Diskussion. Andererseits gehört der Ruf nach einem subjektorientierten Unterricht, der das Individuum in den Mittelpunkt religiösen Lernens stellt, seit vielen Jahrzehnten zum religionspädagogischen Standardprogramm, was in konsequenter Umsetzung zur Thematisierung kontextueller und biographischer Aspekte führen müsste. Die Überführung des Heterogenitätsbegriffs in didaktische Programme für den Religionsunterricht stellt somit ein Desiderat dieser Untersuchung dar. Gleichzeitig kann festgehalten werden, dass in den beobachteten Stunden selbst das Potenzial des Pluralitätsbegriffs kaum ausgeschöpft wurde, weil die stilistische Dimension von Religion eine deutlich geringere Rolle spielt als die positionelle. Bedenkt man, dass es das Ziel aktuellen Religionsunterrichts ist, die Schülerinnen und Schüler zu einem respektvollen Umgang mit religiöser Vielfalt in ihrem Alltag zu befähigen (s. oben), wirft diese Beobachtung die Frage auf, inwiefern eine vor allem positio- 
nelle Aufklärung über Religion(en) zu dieser Kompetenz beiträgt. Zumindest liegt es nahe, dass sich die Pluralitätsfähigkeit der Kinder und Jugendlichen im Alltag vor allem in der Art und Weise bewähren muss, wie Menschen ihre religiösen Orientierungen leben. Letzteres stellen aber stilistische, kontextuell und biographisch rückgebundene Facetten von Religion dar.

Gleichzeitig muss bedacht werden, dass die hier dargestellten Beobachtungen erste, explorative Zugänge zu einem in der Religionsdidaktik erst in jüngster Zeit empirisch adressierten Forschungsfeld darstellen. Sie liefern nur erste Indizien, die durch weitere Projekte bestätigt, differenziert oder korrigiert werden müssen. Generalisierende Aussagen darüber, wie stark bzw. oft religiöse Vielfalt den Inhalt des Religionsunterrichts prägt, können anhand der hier beschriebenen Befunde nicht formuliert werden. Auch lassen die verwendeten Ratingmanuale keinen Schluss darüber zu, inwiefern die Thematisierung besagter Vielfalt zu Diskussionen in der Lerngruppe geführt hat. Auch kann nicht ermessen werden, inwiefern die konfessionelle Rahmung dieses Fachs seinen Umgang mit religiöser Vielfalt bedingt (vgl. Knauth und Vieregge 2018). Derartige Fragestellungen entziehen sich den Erkenntnismöglichkeiten der vorliegenden Untersuchung. Zukünftige Projekte, die derartige Fragestellungen in den Blick nehmen, sollten aber an das vierdimensionale Modell religiöser Vielfalt, wie es sich im vorfindlichen Projekt bewährt hat, anschließen können.

Förderung Die Studie wurde gefördert von der Deutschen Forschungsgemeinschaft (Projektnummer: 401439582).

Funding Open Access funding enabled and organized by Projekt DEAL.

Open Access Dieser Artikel wird unter der Creative Commons Namensnennung 4.0 International Lizenz veröffentlicht, welche die Nutzung, Vervielfältigung, Bearbeitung, Verbreitung und Wiedergabe in jeglichem Medium und Format erlaubt, sofern Sie den/die ursprünglichen Autor(en) und die Quelle ordnungsgemäß nennen, einen Link zur Creative Commons Lizenz beifügen und angeben, ob Änderungen vorgenommen wurden.

Die in diesem Artikel enthaltenen Bilder und sonstiges Drittmaterial unterliegen ebenfalls der genannten Creative Commons Lizenz, sofern sich aus der Abbildungslegende nichts anderes ergibt. Sofern das betreffende Material nicht unter der genannten Creative Commons Lizenz steht und die betreffende Handlung nicht nach gesetzlichen Vorschriften erlaubt ist, ist für die oben aufgeführten Weiterverwendungen des Materials die Einwilligung des jeweiligen Rechteinhabers einzuholen.

Weitere Details zur Lizenz entnehmen Sie bitte der Lizenzinformation auf http://creativecommons.org/ licenses/by/4.0/deed.de.

Interessenkonflikt U. Riegel, M. Gronover, D. Ambiel, M. Brügge-Feldhake, S. Jumpertz, M. Krämer und R. Boschki geben an, dass kein Interessenkonflikt besteht.

\section{Literatur}

Arzt, S. (1999). Frauenwiderstand macht Mädchen Mut: Die geschlechtsspezifische Rezeption einer biblischen Erzählung. Wien: Tyrolia.

Asbrand, B. (2000). Zusammen Leben und Lernen im Religionsunterricht. Frankfurt a. M.: IKO.

Aslan, E. (2017). Die Erziehung muslimischer Kinder zu Pluralitätsfähigkeit. In Y. Sarıkaya \& F.-J. Bäumer (Hrsg.), Aufbruch zu neuen Ufern: Aufgaben, Problemlagen und Profile einer Islamischen Religionspädagogik im europäischen Kontext (S. 15-32). Münster: Waxmann. 
Buchardt, M. (2010). When Muslimness is pedagogized: „Religion“ and „culture“ as knowledge and social classification. British Journal of Religious Education, 32(3), 259-273.

Büttner, G., Mendl, H., Reis, O., \& Roose, H. (Hrsg.). (2018). Heterogenität im Klassenzimmer. Babenhausen: LUSA.

Die Deutschen Bischöfe (Hrsg.). (2016). Die Zukunft des konfessionellen Religionsunterrichts. Bonn: DBK.

Domsgen, M., \& Witten, U. (2018). Religiöse Heterogenität im Religionsunterricht. Worauf wir achten und was verstärkt in den Blick treten sollte. Praktische Theologie, 53(2), 79-83.

Englert, R. (2002a). Dimensionen religiöser Pluralität. In F. Schweitzer, U. Schwab, H.-G. Ziebertz \& R. Englert (Hrsg.), Entwurf einer pluralitätsfähigen Religionspädagogik (S. 17-50). Gütersloh: Gütersloher.

Englert, R. (2002b). Skizze einer pluralitätsfähigen Religionspädagogik. In F. Schweitzer, U. Schwab, H.G. Ziebertz \& R. Englert (Hrsg.), Entwurf einer pluralitätsfähigen Religionspädagogik (S. 87-106). Gütersloh: Gütersloher.

Englert, R. (2015). Connection impossible? Wie konfessioneller Religionsunterricht Schüler/innen ins Gespräch mit Religion bringt. In E.-M. Kenngott, R. Englert \& T. Knauth (Hrsg.), Konfessionell - interreligiös - religionskundlich: Unterrichtsmodelle in der Diskussion (S. 19-30). Stuttgart: Kohlhammer.

Englert, R., Hennecke, E., \& Kämmerling, M. (2014). Innenansichten des Religionsunterrichts: Fallbeispiele - Analysen - Konsequenzen. München: Kösel.

Gärtner, C. (2015). Religionsunterricht - ein Auslaufmodell? Begründungen und Grundlagen religiöser Bildung in der Schule. Paderborn: Schöningh.

Gronover, M. (2012). Konfessionalität in religiöser Heterogenität im Religionsunterricht an berufsbildenden Schulen. In A. Biesinger, F. Schweitzer, M. Gronover \& J. Ruopp (Hrsg.), Integration durch religiöse Bildung. Perspektiven zwischen beruflicher Bildung und Religionspädagogik (S. 173-188). Münster: Waxmann.

Gronover, M., Krause, C., Marose, M., Boschki, R., Meyer-Blank, M., \& Schweitzer F. (Hrsg.) (2021). Offene Konfessionalität. Diskurse mit Expertinnen und Experten zum Profil des Religionsunterrichts an berufsbildenden Schulen. Münster: Waxmann.

Grümme, B. (2014). Bildungsgerechtigkeit: Eine religionspädagogische Herausforderung. Stuttgart: Kohlhammer.

Grümme, B. (2017). Heterogenität in der Religionspädagogik: Grundlagen und konkrete Bausteine. Freiburg: Herder.

Grümme, B. (2018). Auf dem Weg zu einem Paradigmenwechsel in der Religionspädagogik? Skizzen zu einem Konzept Aufgeklärter Heterogenität. Praktische Theologie. https://doi.org/10.14315/prth2018-530204.

Grümme, B. (2021). Umdenken erforderlich? Zur Relevanz von Heterogenität für die Denkform der Religionspädagogik. In B. Grümme, T. Schlag \& N. Ricken (Hrsg.), Heterogenität: Eine Herausforderung für Religionspädagogik und Erziehungswissenschaft (S. 17-27). Stuttgart: Kohlhammer.

Kaupp, A. (2005). Junge Frauen erzählen ihre Glaubensgeschichte. Eine qualitativ-empirische Studie zur Rekonstruktion der narrativen religiösen Identität katholischer junger Frauen. Ostfildern: Schwabenverlag.

Kilchsberger, J.R. (2015). Religionskundliches Lernen: Kulturelle Zugänge und religiöse Konzepte. In S. Bietenhard, D. Helbling \& K. Schmid (Hrsg.), Ethik, Religionen, Gemeinschaft: Ein Studienbuch (S. 203-212). Zürich: hep.

Knauth, T., \& Vieregge, D. (2018). Religious education and dialogue in contextual perspective: a comparative case study in hamburg and Duisburg (Germany). In F. Schweitzer \& R. Boschki (Hrsg.), Researching religious education: classroom processes and outcomes (S. 105-115). Münster: Waxmann.

Könemann, J. (2002). „Ich wünschte, ich wäre gläubig, glaub’ ich. “: Zugänge zu Religion und Religiosität in der Lebensführung der späten Moderne. Wiesbaden: VS.

Kumlehn, M. (2018). Researching approaches to religious education: the example of performative religious education. In F. Schweitzer \& R. Boschki (Hrsg.), Researching religious education: classroom processes and outcomes (S. 89-103). Münster: Waxmann.

Lotz, M., Gabriel, K., \& Lipowsky, F. (2013). Niedrig und hoch inferente Verfahren der Unterrichtsbeobachtung. Analysen zu deren gegenseitiger Validierung. Zeitschrift für Pädagogik, 59(3), 357-380.

Mauritz, G., Hillebrand, M., Reis, O., Wittke, A., \& Kamcili-Yildiz, N. (2020). Mindsets religiöser Pluralität als Faktor in der (islamischen) Religionslehrer*innenbildung. Theo-Web. Zeitschrift für Religionspädagogik, 19(1), 230-248.

Meyer, K. (2019). Grundlagen interreligiösen Lernens. Göttingen: Vandenhoeck \& Ruprecht. 
Owen, S. (2011). The world religions paradigm time for a change. Arts and Humanities in Higher Education, 10(3), 253-268.

Rat der Evangelischen Kirche in Deutschland (Hrsg.). (2014). Religiöse Orientierung gewinnen. Evangelischer Religionsunterricht als Beitrag zu einer pluralitätsfähigen Schule. Gütersloh: Gütersloher.

Reese-Schnitker, A. (2006). „Ich weiß nicht, wo da Religion anfängt und aufhört“. Eine empirische Studie zum Zusammenhang von Lebenswelt und Religiosität bei Singlefrauen. Gütersloh: Gütersloher.

Reis, O. (2021). Unsichtbar, lähmend oder steuernd. Heterogenität als didaktischer Akteur im Unterricht. In B. Grümme, T. Schlag \& N. Ricken (Hrsg.), Heterogenität: Eine Herausforderung für Religionspädagogik und Erziehungswissenschaft (S. 137-150). Stuttgart: Kohlhammer.

Reis, O., Hillebrand, M., Mauritz, G., Wittke, A., \& Kamcili-Yildiz, N. (2020). Mindsets guter Lehre in Beziehung zu den Mindsets religiöser Pluralität. Theo-Web. Zeitschrift für Religionspädagogik, 19(1), 249-266.

Riegel, U. (2004). Gott und Gender: Eine empirisch-religionspädagogische Untersuchung nach Geschlechtsvorstellungen in Gotteskonzepten. Münster: LIT.

Riegel, U. (2018). Video analysis: opening the black box of teaching religious education. In F. Schweitzer \& R. Boschki (Hrsg.), Researching religious education: classroom processes and outcomes (S. 117-129). Münster: Waxmann.

Riegel, U. (2021). Der Religionsunterricht angesichts der öffentlichen Debatte um gesellschaftliche Säkularisierung und Re-Sakralisierung. Zeitschrift für Pädagogik. https://doi.org/10.3262/ZP2101048.

Riegel, U., \& Delling, S. (2019). Dealing with worldviews in religious education. Journal of Beliefs \& Values, 111(8), 1-13.

Sajak, C.P. (2012). Interreligiöses Lernen im schulischen Religionsunterricht. In B. Grümme, H. Lenhard \& M.L. Pirner (Hrsg.), Religionsunterricht neu denken: Innovative Ansätze und Perspektiven der Religionsdidaktik (S. 223-233). Stuttgart: Kohlhammer.

Schweitzer, F. (2002). Religionspädagogische Theoriebildung im Zeichen der Pluralitätsproblematik. In F. Schweitzer, U. Schwab, H.-G. Ziebertz \& R. Englert (Hrsg.), Entwurf einer pluralitätsfähigen Religionspädagogik (S. 75-85). Gütersloh: Gütersloher.

Schweitzer, F. (2021). Religiöser Pluralismus und Heterogenität: konkurrierende, komplementäre oder inkommensurable Grundbegriffe der Religionspädagogik? In B. Grümme, T. Schlag \& N. Ricken (Hrsg.), Heterogenität: Eine Herausforderung für Religionspädagogik und Erziehungswissenschaft (S. 29-39). Stuttgart: Kohlhammer.

Schweitzer, F., Schwab, U., Ziebertz, H.-G., \& Englert, R. (Hrsg.). (2002). Entwurf einer pluralitätsfähigen Religionspädagogik. Gütersloh: Gütersloher.

Schweitzer, F., Wissner, G., Bohner, A., Nowack, R., Gronover, M., \& Boschki, R. (2018). Jugend-Glaube - Religion: Eine Repräsentativstudie zu Jugendlichen im Religions- und Ethikunterricht. Münster: Waxmann.

Seidel, T., Dahlehefte, I., \& Meyer, L. (2005). Standardized guidelines-how to collect videotapes. In T. Seidel, M. Prenzel \& M. Kobarg (Hrsg.), How to run a video study (S. 29-53). Münster: Waxmann.

Simojoki, H. (2020). Faktische Beziehungslosigkeit - grundlegende Zusammengehörigkeit. Zur Pluralitätsverarbeitung in Religionspädagogik und Religionspädagogik. In B. Schröder \& T. Schlag (Hrsg.), Praktische Theologie und Religionspädagogik: Systematische, empirische und thematische Verhältnisbestimmungen (S. 103-118). Leipzig: EVA.

Sorg, P. (2020). Religionsunterricht im globalisierten Klassenzimmer: Positionierungen von Lernenden im multireligiösen Kontext beruflicher Schulen. Religious diversity and education in Europe. Münster: Waxmann.

Trautmann, M., \& Wischer, B. (2011). Heterogenität in der Schule: Eine kritische Einführung. Wiesbaden: VS.

Ulfat, F. (2017). Die Selbstrelationierung muslimischer Kinder zu Gott: Eine empirische Studie über die Gottesbeziehungen muslimischer Kinder als reflexiver Beitrag zur Didaktik des Islamischen Religionsunterrichts. Paderborn: Schöningh.

Unser, A. (2018). Interreligiöses Lernen. In M. Schambeck \& U. Riegel (Hrsg.), Was im Religionsunterricht so läuft. Wege und Ergebnisse religionspädagogischer Unterrichtsforschung (S. 270-285). Freiburg: Herder.

Wagensommer, G., \& Schweitzer, F. (2012). Wertebildung im Religionsunterricht: Eine empirische Untersuchung im berufsbildenden Bereich. Münster: Waxmann.

Wirtz, M. A., \& Caspar, F. (2002). Beurteilerübereinstimmung und Beurteilerreliabilität: Methoden zur Bestimmung und Verbesserung der Zuverlässigkeit von Einschätzungen mittels Kategoriensystemen und Ratingskalen. Berlin: Hogrefe.

Ziebertz, H.-G., Kalbheim, B., Riegel, U., \& Prokopf, A. (2003). Religiöse Signaturen heute: Ein religionspädagogischer Beitrag zur empirischen Jugendforschung. Gütersloh: Gütersloher. 